\title{
A Simplified Descriptor System Approach to Delay-Dependent Stability and Robust Performance Analysis for Discrete-Time Systems with Time Delays
}

\author{
Fengying Xu and Daxin Li \\ Physical Education Department, Qufu Normal University, Qufu, Shandong 273165, China \\ Correspondence should be addressed to Fengying Xu; xufyhanhan@126.com
}

Received 25 November 2012; Accepted 24 February 2013

Academic Editor: Yang Yi

Copyright (C) 2013 F. Xu and D. Li. This is an open access article distributed under the Creative Commons Attribution License, which permits unrestricted use, distribution, and reproduction in any medium, provided the original work is properly cited.

A simplified descriptor system approach is proposed for discrete-time systems with delays in terms of linear matrix inequalities. In comparison with the results obtained by combining the descriptor system approach with recently developed bounding technique, our approach can remove the redundant matrix variables while not reducing the conservatism. It is shown that the bounding technique is unnecessary in the derivation of our results. Via the proposed method, delay-dependent results on quadratic cost and $H_{\infty}$ performance analysis are also presented.

\section{Introduction}

In the past decades, considerable attention has been paid to the problems of stability analysis and control synthesis of time-delay systems. Many methodologies have been proposed, and a large number of results have been established (see, e.g., $[1,2]$ and the references therein). All these results can be generally divided into two categories: delayindependent stability conditions $[3,4]$ and delay-dependent stability conditions [5-11]. The delay-independent stability condition does not take the delay size into consideration and thus is often conservative especially for systems with small delays, while the delay-dependent stability condition makes fully use of the delay information and thus is less conservative than the delay-independent one. Very recently, in order to provide less conservative delay-dependent stability criteria, a descriptor system approach was proposed in $[12,13]$, while a new bounding technique has been presented in [14] (also called Moon's inequality). By combining the descriptor system approach with the bounding technique, novel delaydependent sufficient conditions for the existence of a memoryless feedback guaranteed cost controller are derived for a class of discrete-time systems with delays in $[6,7]$.

Although the descriptor system approach proposed in [12, 13] is powerful to deal with the stability analysis of time-delay systems, there are too many matrix variables introduced. In [15], a simplified but equivalent descriptor system approach to delay-dependent stability analysis was established for the continuous-time systems with delays. It is shown in [15] that the bounding technique in [14] is not necessary when deriving the delay-dependent stability results. It should be pointed out that the result in [15] is only applicable to continuoustime systems with delays. In this paper, we focus our attention upon deriving a simplified descriptor system approach to delay-dependent stability analysis in the context of discretetime systems with delays. It is shown that the results derived by our approach are also equivalent to those obtained in $[6,7]$ but with fewer variables to be determined. It is also proved that, for discrete-time systems, the bounding technique in [14] will introduce some redundant variables and thus is unnecessary. Via the proposed method, delay-dependent results on quadratic cost and $H_{\infty}$ performance analysis are also presented. It is worth mentioning that through the approach proposed in this paper, the delay-dependent guaranteed cost control conditions in $[6,7]$ obtained by the descriptor system approach and the bounding technique can also be simplified.

Notations. Throughout this paper, for real symmetric matrices $X$ and $Y$, the notation $X \geq Y$ (resp., $X>Y$ ) means 
that the matrix $X-Y$ is positive semidefinite (resp., positive definite). The superscript " $T$ " represents the transpose. $I$ is an identity matrix with appropriate dimension. $\operatorname{diag}(\cdot)$ denotes a diagonal matrix. $l_{2}[0, \infty)$ refers to the space of square summable infinite vector sequences. In symmetric block matrices, we use an asterisk “*” to represent a term that is induced by symmetry. Matrices, if not explicitly stated, are assumed to have compatible dimensions for algebraic operations.

\section{Main Results}

In order to introduce the simplified descriptor system approach, we consider the following discrete time-delay system

$$
\begin{aligned}
\left(\Sigma_{a}\right): x(k+1) & =\sum_{i=0}^{2} A_{i} x\left(k-d_{i}(k)\right), \\
x(k) & =\phi(k), \quad \forall k \in[-h, 0],
\end{aligned}
$$

where $x(k) \in \mathbb{R}^{n}$ is the state, $d_{0}(k)=0, \phi(k)$ is the initial condition, the scalar $h>0$ is an upper bound on the time delays $d_{i}(k), i=1,2$, and $A_{i}, i=0,1,2$, are known real constant matrices. tion.

Throughout this paper, we make the following assump-

Assumption 1. $d_{i}(k)$ are unknown but satisfy for all $k \in \mathbb{Z}^{+}$

$$
0<d_{i}(k) \leq d_{i}, \quad i=1,2 .
$$

Now, we are in a position to present the main result of this paper.

Theorem 2. Under Assumption 1, the time-delay system $\left(\Sigma_{a}\right)$ is asymptotically stable for all $d_{i}(k), i=1,2$, satisfying (2) if there exist matrices $P>0, P_{i}, R_{i}, S_{i}$, and $L_{i}, i=1,2$, such that the following LMI holds:

$$
\left[\begin{array}{cccccc}
\Omega & G^{T} & {\left[\begin{array}{c}
0 \\
A_{1}
\end{array}\right]-L_{1}^{T}} & G^{T}\left[\begin{array}{c}
0 \\
A_{2}
\end{array}\right]-L_{2}^{T} & -d_{1} L_{1}^{T} & -d_{2} L_{2}^{T} \\
* & -R_{1} & 0 & 0 & 0 \\
* & * & -R_{2} & 0 & 0 \\
* & * & * & -d_{1} S_{1} & 0 \\
* & * & * & * & -d_{2} S_{2}
\end{array}\right]<0,
$$

where

$$
\begin{aligned}
G= & {\left[\begin{array}{cc}
P & 0 \\
P_{1} & P_{2}
\end{array}\right], } \\
\Omega= & G^{T}\left[\begin{array}{cc}
0 & I \\
A_{0}-I & -I
\end{array}\right]+\left[\begin{array}{cc}
0 & I \\
A_{0}-I & -I
\end{array}\right]^{T} G+\sum_{i=1}^{2}\left[\begin{array}{c}
L_{i} \\
0
\end{array}\right] \\
& +\sum_{i=1}^{2}\left[\begin{array}{c}
L_{i} \\
0
\end{array}\right]^{T}+\left[\begin{array}{cc}
\sum_{i=1}^{2} R_{i} & 0 \\
0 & P+\sum_{i=1}^{2} d_{i} S_{i}
\end{array}\right] .
\end{aligned}
$$

Proof. For all $d_{i}(k), i=1,2$, satisfying (2), it can be verified that (3) implies that

$\Theta(k)$

$:=\left[\begin{array}{ccccc}\Omega & G^{T}\left[\begin{array}{c}0 \\ A_{1}\end{array}\right]-L_{1}^{T} & G^{T}\left[\begin{array}{c}0 \\ A_{2}\end{array}\right]-L_{2}^{T} & -d_{1}(k) L_{1}^{T} & -d_{2}(k) L_{2}^{T} \\ * & -R_{1} & 0 & 0 & 0 \\ * & * & -R_{2} & 0 & 0 \\ * & * & * & -d_{1}(k) S_{1} & 0 \\ * & * & * & * & -d_{2}(k) S_{2}\end{array}\right]$

$<0$

Let

$$
y(k)=x(k+1)-x(k)
$$

It is easy to see that

$$
x\left(k-d_{i}(k)\right)=x(k)-\sum_{l=k-d_{i}(k)}^{k-1} y(l) .
$$

Then, the system $\left(\Sigma_{a}\right)$ can be transformed into an equivalent descriptor form

$$
\begin{gathered}
x(k+1)=x(k)+y(k) \\
0=-y(k)+\left(\sum_{i=0}^{2} A_{i}-I\right) x(k)-\sum_{i=1}^{2} A_{i}\left(\sum_{l=k-d_{i}(k)}^{k-1} y(l)\right) .
\end{gathered}
$$

Now, choose a Lyapunov functional candidate as

$$
V(k)=V_{1}(k)+V_{2}(k)+V_{3}(k),
$$

where

$$
\begin{aligned}
& V_{1}(k)=x^{T}(k) P x(k), \\
& V_{2}(k)=\sum_{i=1}^{2}\left\{\sum_{l=k-d_{i}(k)}^{k-1} x^{T}(l) R_{i} x(l)\right\}, \\
& V_{3}(k)=\sum_{i=1}^{2}\left\{\sum_{\theta=-d_{i}(k)+1}^{0} \sum_{l=k-1+\theta}^{k-1} y^{T}(l) S_{i} y(l)\right\} .
\end{aligned}
$$


Then,

$$
\begin{aligned}
& \Delta V_{1}(k) \\
& =V_{1}(k+1)-V_{1}(k) \\
& =2 x^{T}(k) P y(k)+y^{T}(k) P y(k) \\
& =2 \bar{x}^{T}(k) G^{T}\left[\begin{array}{c}
y(k) \\
0
\end{array}\right]+y^{T}(k) P y(k) \\
& =2 \bar{x}^{T}(k) G^{T} \\
& \times\left[-y(k)+\left(\sum_{i=0}^{2} A_{i}-I\right) x(k)-\sum_{i=1}^{2} A_{i}\left(\sum_{l=k-d_{i}(k)}^{k-1} y(l)\right)\right] \\
& +y^{T}(k) P y(k) \\
& =2 \bar{x}^{T}(k) G^{T}\left[\begin{array}{cc}
0 & I \\
\sum_{i=0}^{2} A_{i}-I & -I
\end{array}\right] \bar{x}(k) \\
& +\bar{x}^{T}(k)\left[\begin{array}{ll}
0 & 0 \\
0 & P
\end{array}\right] \bar{x}(k) \\
& -2 \bar{x}^{T}(k) \sum_{i=1}^{2} G^{T}\left[\begin{array}{c}
0 \\
A_{i}
\end{array}\right] \sum_{l=k-d_{i}(k)}^{k-1} y(l) \\
& =2 \bar{x}^{T}(k) G^{T}\left[\begin{array}{cc}
0 & I \\
\sum_{i=0}^{2} A_{i}-I & -I
\end{array}\right] \bar{x}(k) \\
& +\bar{x}^{T}(k)\left[\begin{array}{ll}
0 & 0 \\
0 & P
\end{array}\right] \bar{x}(k) \\
& +2 \bar{x}^{T}(k) \sum_{i=1}^{2}\left(L_{i}^{T}-G^{T}\left[\begin{array}{c}
0 \\
A_{i}
\end{array}\right]\right) \sum_{l=k-d_{i}(k)}^{k-1} y(l) \\
& -2 \bar{x}^{T}(k) \sum_{i=1}^{2} L_{i}^{T} \sum_{l=k-d_{i}(k)}^{k-1} y(l) \\
& =2 \bar{x}^{T}(k) G^{T}\left[\begin{array}{cc}
0 & I \\
\sum_{i=0}^{2} A_{i}-I & -I
\end{array}\right] \bar{x}(k)+\bar{x}^{T}(k)\left[\begin{array}{ll}
0 & 0 \\
0 & P
\end{array}\right] \bar{x}(k) \\
& +2 \bar{x}^{T}(k) \sum_{i=1}^{2}\left(L_{i}^{T}-G^{T}\left[\begin{array}{c}
0 \\
A_{i}
\end{array}\right]\right)\left[x(k)-x\left(k-d_{i}(k)\right)\right] \\
& -2 \bar{x}^{T}(k) \sum_{i=1}^{2} L_{i}^{T} \sum_{l=k-d_{i}(k)}^{k-1} y(l) \\
& =2 \bar{x}^{T}(k)\left(G^{T}\left[\begin{array}{cc}
0 & I \\
A_{0}-I & -I
\end{array}\right]+\sum_{i=1}^{2}\left[\begin{array}{c}
L_{i} \\
0
\end{array}\right]^{T}\right) \bar{x}(k) \\
& +\bar{x}^{T}(k)\left[\begin{array}{ll}
0 & 0 \\
0 & P
\end{array}\right] \bar{x}(k)
\end{aligned}
$$

$$
\begin{aligned}
& +2 \bar{x}^{T}(k) \sum_{i=1}^{2}\left(G^{T}\left[\begin{array}{c}
0 \\
A_{i}
\end{array}\right]-L_{i}^{T}\right) x\left(k-d_{i}(k)\right) \\
& -2 \bar{x}^{T}(k) \sum_{i=1}^{2} L_{i}^{T} \sum_{l=k-d_{i}(k)}^{k-1} y(l)
\end{aligned}
$$

where $\bar{x}(k)=\left[\begin{array}{ll}x^{T}(k) & y^{T}(k)\end{array}\right]^{T}$.

Furthermore, from (11), we obtain

$$
\begin{aligned}
& \Delta V_{1}(k) \\
& =\frac{1}{d_{1}(k) d_{2}(k)} \\
& \times \sum_{\alpha_{2}=k-d_{2}(k)}^{k-1} \sum_{\alpha_{1}=k-d_{1}(k)}^{k-1}\left[2 \bar{x}^{T}(k)\right. \\
& \times\left(G^{T}\left[\begin{array}{cc}
0 & I \\
A_{0}-I & -I
\end{array}\right]\right. \\
& \left.+\sum_{i=1}^{2}\left[\begin{array}{c}
L_{i} \\
0
\end{array}\right]^{T}+\left[\begin{array}{cc}
0 & 0 \\
0 & \frac{1}{2} P
\end{array}\right]\right) \bar{x}(k) \\
& +2 \bar{x}^{T}(k) \sum_{i=1}^{2}\left(G^{T}\left[\begin{array}{c}
0 \\
A_{i}
\end{array}\right]-L_{i}^{T}\right) \\
& \times x\left(k-d_{i}(k)\right) \\
& -2 d_{1}(k) \bar{x}^{T}(k) L_{1}^{T} y\left(\alpha_{1}\right) \\
& \left.-2 d_{2}(k) \bar{x}^{T}(k) L_{2}^{T} y\left(\alpha_{2}\right)\right] .
\end{aligned}
$$

After some manipulations, we get

$$
\begin{aligned}
& \Delta V_{2}(k)+ \Delta V_{3}(k) \\
& \leq \sum_{i=1}^{2}\left[x^{T}(k) R_{i} x(k)+d_{i} y^{T}(k) S_{i} y(k)\right. \\
& \\
& \quad-x^{T}\left(k-d_{i}(k)\right) R_{i} x\left(k-d_{i}(k)\right) \\
&\left.\quad-\sum_{l=k-d_{i}(k)}^{k-1} y^{T}(l) S_{i} y(l)\right] \\
&=\frac{1}{d_{1}(k) d_{2}(k)}
\end{aligned}
$$




$$
\begin{aligned}
\times \sum_{\alpha_{2}=k-d_{2}(k)}^{k-1} \sum_{\alpha_{1}=k-d_{1}(k)}^{k-1}\left\{\bar{x}^{T}(k)\right. & {\left[\begin{array}{cc}
\sum_{i=1}^{2} R_{i} & 0 \\
0 & \sum_{i=1}^{2} d_{i} S_{i}
\end{array}\right] \bar{x}(k) } \\
& -d_{2}(k) y^{T}\left(\alpha_{2}\right) S_{2} y\left(\alpha_{2}\right) \\
& -d_{1}(k) y^{T}\left(\alpha_{1}\right) S_{1} y\left(\alpha_{1}\right) \\
& -\sum_{i=1}^{2} x^{T}\left(k-d_{i}(k)\right) \\
& \left.\times R_{i} x\left(k-d_{i}(k)\right)\right\} .
\end{aligned}
$$

Combining (12) with (13) yields

$\Delta V(k)$

$$
\begin{aligned}
\leq & \frac{1}{d_{1}(k) d_{2}(k)} \\
& \times \sum_{\alpha_{2}=k-d_{2}(k)}^{k-1} \sum_{\alpha_{1}=k-d_{1}(k)}^{k-1} \eta^{T}\left(k, \alpha_{1}, \alpha_{2}\right) \Theta(k) \eta\left(k, \alpha_{1}, \alpha_{2}\right),
\end{aligned}
$$

where $\Theta(k)$ is given in (5) and

$$
\begin{aligned}
& \eta\left(k, \alpha_{1}, \alpha_{2}\right) \\
& =\left[\begin{array}{llllll}
\bar{x}^{T}(k) & x^{T}\left(k-d_{1}(k)\right) & x^{T}\left(k-d_{2}(k)\right) & y^{T}\left(\alpha_{1}\right) & y^{T}\left(\alpha_{2}\right)
\end{array}\right]^{T} .
\end{aligned}
$$

Therefore, the time-delay system $\left(\Sigma_{a}\right)$ is asymptotically stable for all $d_{i}(k), i=1,2$, satisfying (2) by the Lyapunov stability theory. This completes the proof.

Remark 3. It is noted that only two time delays are considered for the sake of simplicity. However, the results in Theorem 2 can be extended to the case of multiple delays. The simplified approach in Theorem 2 can also be used to tackle with the discrete time-delay systems with uncertainties, such as normbounded parameter uncertainties and linear fractional uncertainties.

Remark 4. Note that the delays considered here satisfy (2). From the proof of Theorem 2, the delay-dependent results in this paper can be extended to the case of interval delays (see
TABLE 1: the maximum delay bound of $d_{1}$.

\begin{tabular}{lcccc}
\hline References & {$[18]$} & {$[17]$} & {$[6]$} & Theorem 2 \\
\hline$d_{1}$ & - & 12 & 16 & 18 \\
\hline
\end{tabular}

[16] for more details), where the delays vary between a lower bound (may be not zero) and an upper bound.

By the method proposed in Theorem 2, the quadratic cost analysis result derived by using the descriptor system approach, together with the inequality in [14] as shown in $[6,7]$, can also be simplified. To make it clear, introduce the following quadratic cost function

$$
J=\sum_{k=0}^{\infty} x^{T}(k) Q x(k)
$$

Then, by Theorem 2, we have the following result.

Theorem 5. There exist matrices $P>0, P_{i}, R_{i}, S_{i}$, and $L_{i}, i=$ 1,2 , such that the following LMI holds:

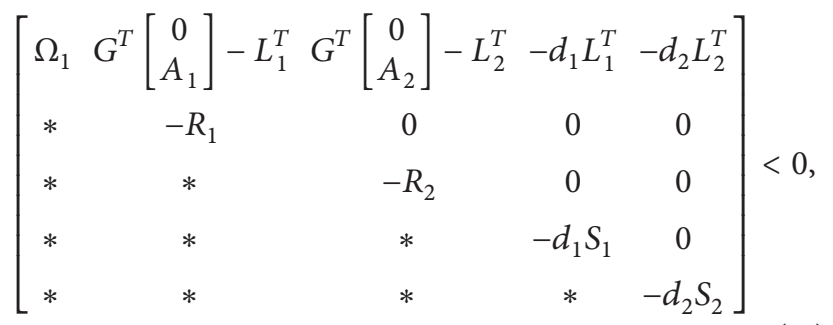

where $\Omega_{1}=\Omega+\operatorname{diag}(Q, 0)$, with $G$ and $\Omega$ being defined in (4), then the system $\left(\Sigma_{a}\right)$ is asymptotically stable, and the cost function in (16) satisfies

$$
\begin{aligned}
J \leq & J_{0} \\
= & x^{T}(0) P x(0) \\
& +\sum_{i=1}^{2}\left\{\sum_{l=-d_{i}}^{-1} x^{T}(l) R_{i} x(l)+\sum_{\theta=-d_{i}+1}^{0} \sum_{l=-1+\theta}^{-1} y^{T}(l) S_{i} y(l)\right\},
\end{aligned}
$$

where $y(l)=x(l+1)-x(l)$.

In the next, via the method proposed in Theorem 2, we will present the $H_{\infty}$ performance analysis result.

Consider the following time-delay system:

$$
\begin{aligned}
\left(\Sigma_{b}\right): x(k+1) & =\sum_{i=0}^{2} A_{i} x\left(k-d_{i}(k)\right)+B \omega(k), \\
z(k) & =C x(k)+D \omega(k),
\end{aligned}
$$

where $z(k) \in \mathbb{R}^{p}$ is the output and $\omega(k) \in \mathbb{R}^{q}$ is the disturbance signal which is assumed to be in $l_{2}[0, \infty)$.

Then, the following delay-dependent result on $H_{\infty}$ performance analysis can be obtained by Theorem 2 . 
Theorem 6. Given a scalar $\gamma>0$. Then, under Assumption 1, the time-delay system $\left(\Sigma_{b}\right)$ :

(i) is asymptotically stable with $\omega(k)=0$,

(ii) satisfies under zero-initial condition for all nonzero $\omega \in l_{2}[0, \infty)$ if there exist matrices $P>0, P_{i}, R_{i}>0, S_{i}>0, L_{i}, i=1,2$, such that the following LMI holds:

$$
\|z\|_{2}<\gamma\|\omega\|_{2}
$$

$$
\left[\begin{array}{cccccccc}
\Omega_{2} & G^{T} & {\left[\begin{array}{c}
0 \\
A_{1}
\end{array}\right]-L_{1}^{T}} & G^{T}\left[\begin{array}{c}
0 \\
A_{2}
\end{array}\right]-L_{2}^{T} & -d_{1} L_{1}^{T} & -d_{2} L_{2}^{T} & G^{T}\left[\begin{array}{c}
0 \\
B
\end{array}\right] & {\left[\begin{array}{c}
C^{T} \\
0
\end{array}\right]} \\
* & -R_{1} & 0 & 0 & 0 & 0 & 0 \\
* & * & -R_{2} & 0 & 0 & 0 & 0 \\
* & * & * & -d_{1} S_{1} & 0 & 0 & 0 \\
* & * & * & * & -d_{2} S_{2} & 0 & 0 \\
* & * & * & * & * & -\gamma I & D^{T} \\
* & * & * & * & * & * & -\gamma I
\end{array}\right]<0,
$$

where $\Omega_{2}=\Omega+\operatorname{diag}\left(C^{T} C, 0\right)$, with $G$ and $\Omega$ being defined in (4).

\section{A Numerical Example}

In this section, we present a numerical example to the effectiveness of the proposed algorithm. In order to show the comparison,we choose $A_{2}=0$ and $d_{2}=0$.

Example 7. Consider the system $\left(\Sigma_{a}\right)$ with

$$
A_{0}=\left[\begin{array}{cc}
0.8 & 0 \\
0 & 0.97
\end{array}\right], \quad A_{1}=\left[\begin{array}{cc}
-0.1 & 0 \\
-0.1 & -0.1
\end{array}\right]
$$

Based on Theorem 2, we seek the maximum value of $d_{1}$. Compared with three methods, which are in $[6,17,18]$, respectively; we can illustrate the advantage of the proposed algorithm in this paper. Table 1 presents the result of comparison.

\section{Conclusions}

In this paper, we have proposed a simplified delay-dependent stability condition for discrete-time systems with delays. The given condition has fewer variables compared with those established using the descriptor system approach with Moon's bounding technique. It has been shown that Moon's bounding technique is unnecessary when deriving the delay-dependent stability conditions. By the proposed method in this paper, the delay-dependent results on quadratic cost and $H_{\infty}$ performance analysis have also been provided.

\section{Acknowledgment}

This work was supported by the Specialized Research Fund for the Doctoral Program of Higher Education under Grant 20113705120003.

\section{References}

[1] J. Hale, Theory of Functional Differential Equations, Springer, New York, NY, USA, 2nd edition, 1977.

[2] Q.-L. Han, "Robust stability of uncertain delay-differential systems of neutral type," Automatica, vol. 38, no. 4, pp. 719-723, 2002.

[3] M. S. Mahmoud and N. F. Al-Muthairi, "Quadratic stabilization of continuous time systems with state-delay and norm-bounded time-varying uncertainties," IEEE Transactions on Automatic Control, vol. 39, no. 10, pp. 2135-2139, 1994.

[4] J. H. Lee, S. W. Kim, and W. H. Kwon, "Memoryless $H_{\infty}$ controllers for state delayed systems," IEEE Transactions on Automatic Control, vol. 39, no. 1, pp. 159-162, 1994.

[5] S. Xu, J. Lam, and C. Yang, " $H_{\infty}$ and positive-real control for linear neutral delay systems," IEEE Transactions on Automatic Control, vol. 46, no. 8, pp. 1321-1326, 2001.

[6] W. Chen, Z. Guan, and X. Lu, "Delay-dependent guaranteed cost control for uncertain discretetime systems with delay," IEE Proceedings Control Theory \& Applications, vol. 150, no. 4, pp. 412-416, 2003.

[7] W.-H. Chen, Z.-H. Guan, and X. Lu, "Delay-dependent guaranteed cost control for uncertain discrete-time systems with both state and input delays," Journal of the Franklin Institute, vol. 341, no. 5, pp. 419-430, 2004.

[8] G. Zong, L. Hou, and J. Li, "A descriptor system approach to $l_{2}-l_{\infty}$ filtering for uncertain discrete-time switched system with mode-dependent time-varying delays," International Journal of Innovative Computing, Information and Control, vol. 7, no. 5, pp. 2213-2224, 2011.

[9] G. Zong, L. Hou, and Y. Wu, "Exponential $l_{2}-l_{\infty}$ filtering for discrete-time switched systems under a new framework," International Journal of Adaptive Control and Signal Processing, vol. 26, no. 2, pp. 124-137, 2012.

[10] T. Li, L. Guo, and X. Xin, "Improved delay-dependent bounded real lemma for uncertain time-delay systems," Information Sciences, vol. 179, no. 20, pp. 3711-3719, 2009.

[11] T. Li, L. Guo, and L. Wu, "Simplified approach to the asymptotical stability of linear systems with interval time-varying delay," IET Control Theory \& Applications, vol. 3, no. 2, pp. 252-260, 2009. 
[12] E. Fridman, "New Lyapunov-Krasovskii functionals for stability of linear retarded and neutral type systems," Systems \& Control Letters, vol. 43, no. 4, pp. 309-319, 2001.

[13] E. Fridman and U. Shaked, "A descriptor system approach to $H_{\infty}$ control of linear time-delay systems," IEEE Transactions on Automatic Control, vol. 47, no. 2, pp. 253-270, 2002.

[14] Y. S. Moon, P. Park, W. H. Kwon, and Y. S. Lee, "Delay-dependent robust stabilization of uncertain state-delayed systems," International Journal of Control, vol. 74, no. 14, pp. 1447-1455, 2001.

[15] S. Xu, J. Lam, and Y. Zou, "Simplified descriptor system approach to delay-dependent stability and performance analysis for time-delay systems," IEE Proceedings Control Theory \& Applications, vol. 152, no. 2, pp. 17-151, 2005.

[16] S. Xu and T. Chen, "Robust $H_{\infty}$ control for uncertain discretetime systems with time-varying delays via exponential output feedback controllers," Systems \& Control Letters, vol. 51, no. 3-4, pp. 171-183, 2004.

[17] Y. S. Lee and W. H. Kwon, "Delay-Dependent robust stabilization of uncertain discrete-time state-delayed systems," in Proceedings of the 15th IFAC Congress on Automation and Control, Barcelona, Spain, 2002.

[18] S.-H. Song, J.-K. Kim, C.-H. Yim, and H.-C. Kim, " $H_{\infty}$ control of discrete-time linear systems with time-varying delays in state," Automatica, vol. 35, no. 9, pp. 1587-1591, 1999. 


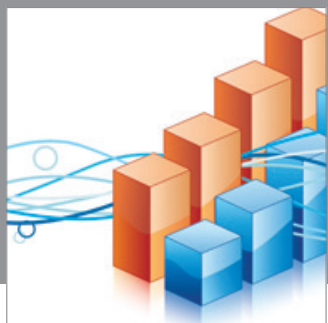

Advances in

Operations Research

mansans

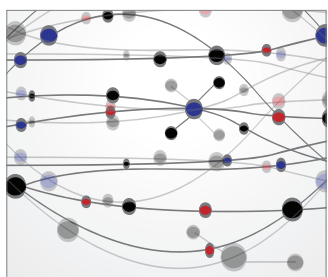

The Scientific World Journal
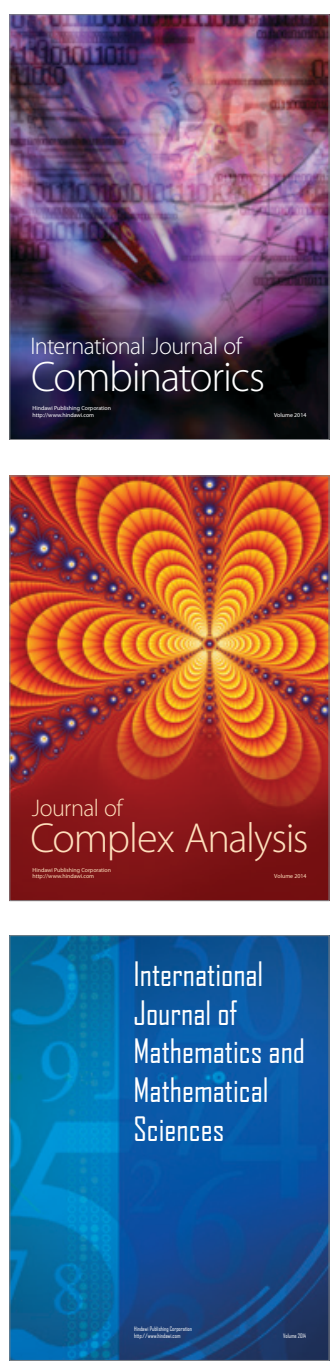
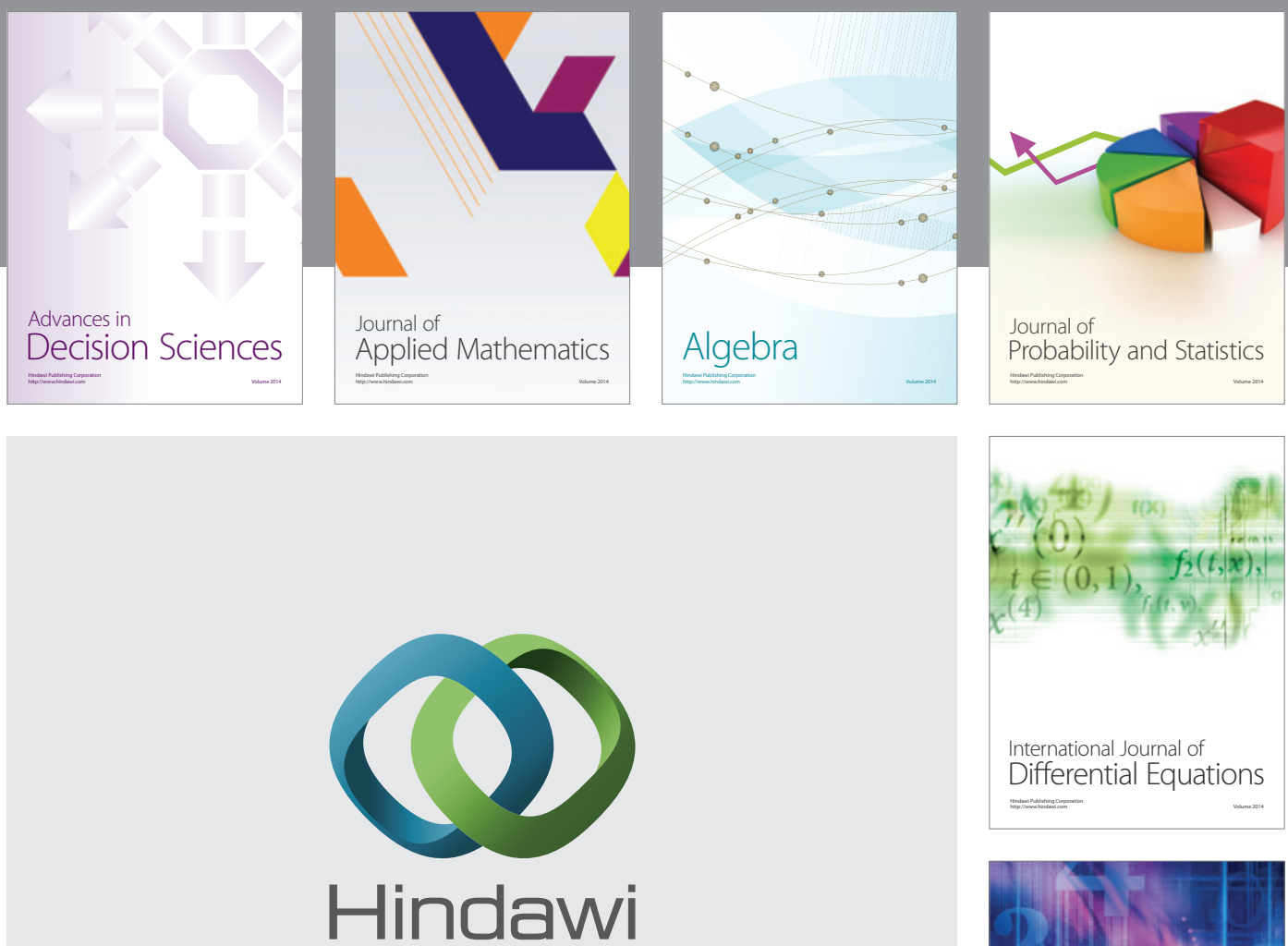

Submit your manuscripts at http://www.hindawi.com
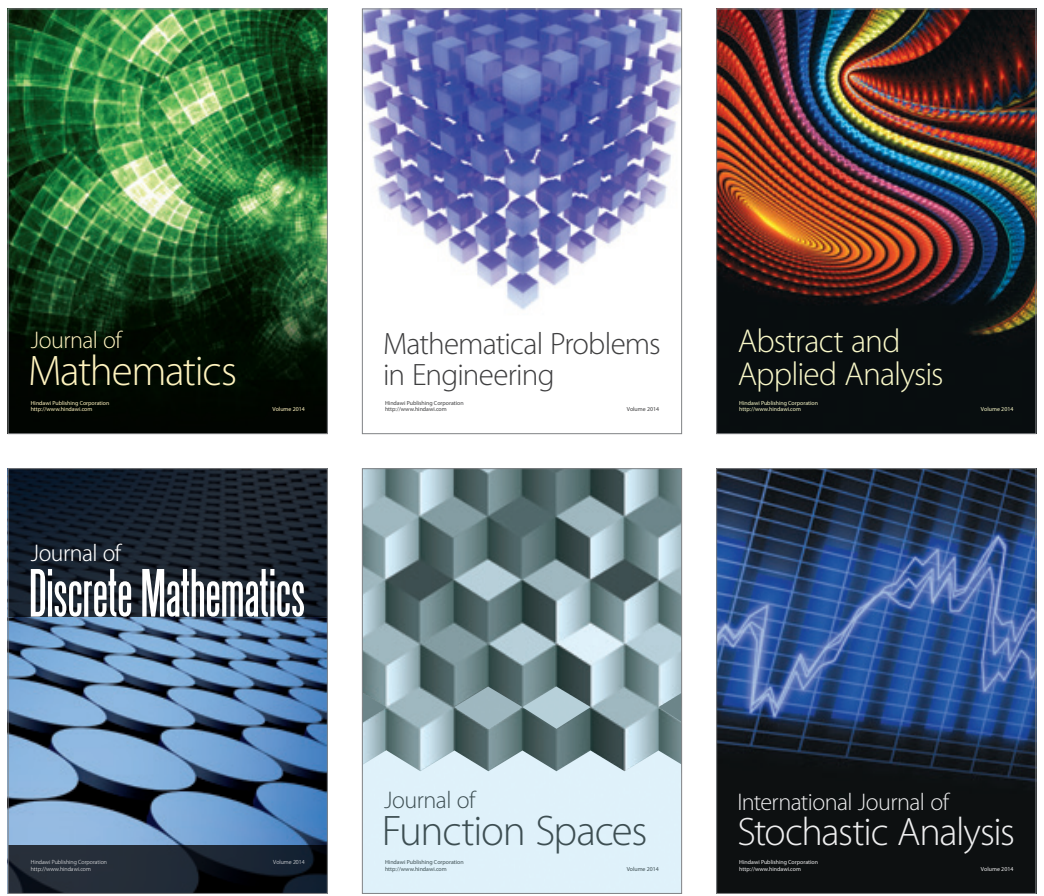

Journal of

Function Spaces

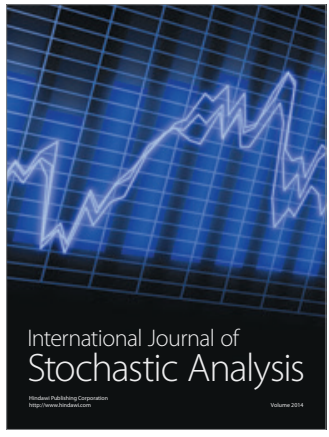

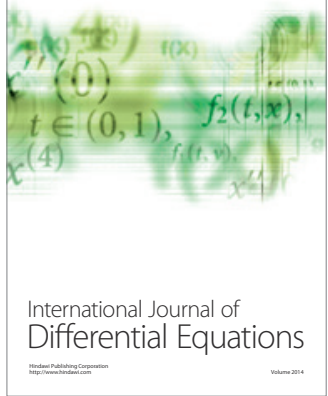
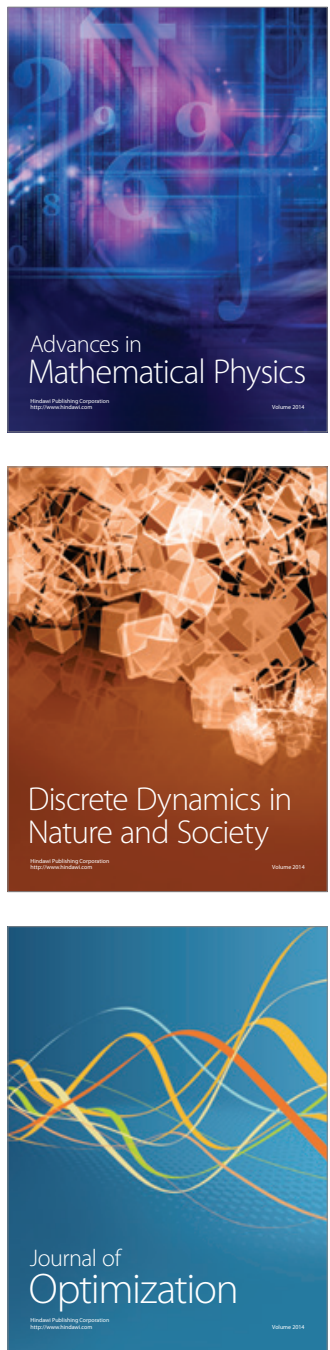